\title{
Enfermeiros na operacionalização do Kanban: novos sentidos para a prática profissional em contexto hospitalar?
}

\author{
Nurses in the Kanban: are there news meanings of professional \\ practice in innovative tools for hospital care management?
}

\author{
Luiz Carlos de Oliveira Cecílio (https://orcid.org/0000-0002-9207-4781) ${ }^{1}$ \\ Ademar Arthur Chioro dos Reis (https://orcid.org/0000-0001-7184-2342) ${ }^{1}$ \\ Rosemarie Andreazza (https://orcid.org/0000-0002-3332-2183) ${ }^{1}$ \\ Sandra Maria Spedo (https://orcid.org/0000-0001-5951-5314) ${ }^{1}$ \\ Nelma Lourenço de Matos Cruz (https://orcid.org/0000-0003-0451-9822) ${ }^{1}$ \\ Luciana Soares de Barros (https://orcid.org/0000-0001-6320-4308) ${ }^{1}$ \\ Graça Carapinheiro (https://orcid.org/0000-0003-4374-5021) ${ }^{3}$ \\ Tiago Correia (https://orcid.org/0000-0001-6015-3314) 2,3 \\ Mariana Cabral Schveitzer (https://orcid.org/0000-0001-9833-2932) ${ }^{1}$
}

${ }^{1}$ Departamento de Medicina Preventiva. Escola Paulista de Medicina. Universidade Federal de São Paulo. R. Botucatu 740, Vila Clementino. 04023-062 São Paulo SP Brasil. luizcecilio60@gmail.com ${ }^{2}$ Unidade de Saúde Pública Internacional e Bioestatística. Instituto de Higiene e Medicina Tropical. Universidade Nova de Lisboa. Lisboa Portugal.

${ }^{3}$ Centro de Investigação

e Estudos em Sociologia. Instituto Universitário de Lisboa. Lisboa Portugal.

\begin{abstract}
Kanban is a care management tool that values multi-professional work and intensive use of data and has been growingly used in Brazil to address overcrowding in hospital emergency services (HES). The researchers monitored the Kanban for ten months in multiple wards of a municipal HES, and their observations were recorded in field diaries and discussed in biweekly research team meetings. The empirical material was organized from two questions: Are there changes in "traditional attributions" of Kanban-operating nursing? Are Medicine-Nursing interprofessional relationships transformed? A strong nurse adherence to this tool was observed, coupled with greater specialization and fragmentation of their work: nurses working as diarists assume more traditional administrative functions, while those on-call develop more direct assistance to patients. Nurses consider that clinical decisions are still in the doctors' hands, although Kanban provides them with a stronger influence on such decisions. Nurses' role in the management of significant mass of clinical and operational data, central to Kanban's operationalization, strengthens their professional authority.
\end{abstract}

Key words Nursing, Emergency medical services, Patient-centered care, Kanban, Medicine-Nursing relationships
Resumo O Kanban, uma tecnologia de gestão do cuidado caracterizada pela valorização do trabalho multiprofissional e o uso intensivo da informação, tem sido crescentemente utilizado para enfrentar a superlotação dos serviços de emergência hospitalar (SEH). Pesquisadores acompanharam durante dez meses o Kanban em vários setores de um SEH municipal. Suas observações, registradas em diários de campo, foram discutidos em reuniões quinzenais por um coletivo de pesquisadores. O material empírico foi organizado a partir de duas questões: Há mudanças nas "atribuições tradicionais" da enfermagem atuando no Kanban? Há transformações nas relações interprofissionais entre Medicina e Enfermagem na operacionalização do Kanban? Observou-se forte adesão dos enfermeiros ao arranjo, pari passu com uma maior especialização e fragmentação do seu trabalho. Os diaristas assumem funções administrativas tradicionais da enfermagem, enquanto os plantonistas desenvolvem assistência direta aos pacientes. Os enfermeiros consideram que a decisão clínica ainda é do médico, embora o Kanban propicie sua maior influência na decisão. A gestão de grande massa de dados clínico-operacionais pelos enfermeiros, central na operacionalização do Kanban, reforça sua autoridade profissional.

Palavras-chave Enfermagem, Serviços médicos de emergência, Assistência Centrada no Paciente, Kanban, Relações Médico-Enfermeiro 


\section{Introdução}

Fenômenos de demanda excessiva, como a superlotação e queixas relativas à qualidade do cuidado em saúde manifestam-se em diversos pontos de atenção dos sistemas de saúde. É nos serviços hospitalares de emergência (SHE), todavia, que se observa maior insatisfação dos usuários, seja pelo tempo de espera para atendimento, seja pela indisponibilidade de leitos hospitalares para internações, acarretando a permanência de um grande número de pacientes por longos períodos em macas nos corredores. Uma das consequências dessas situações é o aumento dos índices de adoecimento das equipes de trabalho, em função de ambientes e processos de trabalho estressores $^{1-5}$.

Neste cenário, a experimentação de intervenções que procuram melhorar a capacidade de acolher, identificar riscos, ofertar cuidados em saúde e gerar saídas nos SHE adquirem grande importância ${ }^{4,6-8}$. Para tanto, os hospitais têm experimentado novos modos de fazer a gestão do cuidado, tanto buscando racionalizar a utilização dos recursos, em particular a diminuição da média de permanência e ocupação plena de sua capacidade instalada, como a qualificação do cuidado, com valorização do trabalho multiprofissional e interdisciplinar, protocolizações e uso intensivo de indicadores para acompanhamento e avaliação do seu sucesso9 ${ }^{9-11}$.

Para enfrentar tais desafios, diversos arranjos tecnológicos para a coordenação do cuidado têm sido utilizados no Brasil, e passaram a ser induzidos pelo Ministério da Saúde, em especial a partir da Política Nacional de Atenção Hospitalar ${ }^{12}$.

Arranjos tecnológicos de cuidado são um conjunto de tecnologias, melhores práticas e instâncias de gestão que, aplicadas preferencialmente de forma combinada, constituem-se em modalidades de intervenção destinadas à aplicação do conhecimento científico para fins práticos na gestão e produção do cuidado em saúde 10,13-18. $^{1}$.

Entre os vários dispositivos utilizados para intervenções voltadas ao aprimoramento dos processos de produção do cuidado e de gestão dos SHE, pode-se destacar o "Kanban", implantado em diferentes serviços sob distintos formatos ${ }^{19-22}$.

O Kanban é um dispositivo para gestão de leitos que combina práticas de gestão de prioridades clínicas e ferramentas visuais que permitem a qualificação da coordenação do cuidado, através da priorização e do aperfeiçoamento da tomada de decisão clínica e da maximização do uso dos leitos, com forte ênfase em equipes mul- tiprofissionais. Sua ação tem sido potencializada pela institucionalização da horizontalidade do cuidado, isso é, a presença cotidiana de profissionais médicos e enfermeiros para uma melhor coordenação da decisão clínica e a continuidade do cuidado. O Kanban faz uso intensivo de informação produzida e utilizada pelas equipes em tempo real, bem como de protocolos clínicos hospitalares ${ }^{15,20,22,23}$.

Pouco se tem abordado possíveis impactos e reconfigurações do Kanban nas práticas de profissionais de saúde e nas relações interprofissionais. O presente artigo segue a linha da literatura internacional que atribui destaque à enfermagem de prática avançada, ou nurse practitioner, nos serviços de emergência ${ }^{24,25}$. O que se sabe é que a enfermagem de prática avançada pode melhorar o acesso, diminuir a lotação, melhorar a qualidade do cuidado, diminuir o tempo de permanência e aumentar da satisfação do paciente ${ }^{26,27}$.

O que falta saber são as possíveis transformações que o Kanban pode produzir na profissão de enfermagem: 1) se há mudanças nas "atribuições tradicionais" da enfermagem na divisão do trabalho; e 2) se há transformações nas relações com a medicina na operacionalização deste arranjo.

\section{Material e métodos}

O artigo é parte de uma investigação maior que teve como objetivo geral compreender como arranjos de gestão do cuidado em SHE são operacionalizados pelos profissionais. Realizou-se uma pesquisa de caráter qualitativo, tipo estudo de caso $^{28,29}$.

O estudo foi conduzido em um Hospital de Urgência Emergência (HUE), pertencente a uma rede municipal de saúde. Trata-se de uma unidade hospitalar certificada como hospital de ensino, referência para o atendimento de urgência e emergência, atendendo tanto à demanda espontânea, como a referenciada pelo SAMU e pelas UPAs do município. Arranjos tecnológicos propostos pela Política Nacional de Atenção Hospitalar do Ministério da Saúde ${ }^{12}$, como o acolhimento com classificação de risco, a gestão de leito e de cuidado (Kanban) estão implantados no hospital.

O Kanban foi eleito para as reflexões que se pretende fazer nesse texto pelo papel central dos enfermeiros na "condução" do arranjo. No Quadro 1 estão descritas as principais características dos Kanbans realizados no HUE.

As estratégias metodológicas adotadas, de orientação etnográfica, traduziram-se na presen- 
Quadro 1. Caracterização do dispositivo Kanban.

\begin{tabular}{|c|c|c|c|c|}
\hline Denominação & Local & Descrição & Periodicidade & Profissionais \\
\hline $\begin{array}{l}\text { Kanban da } \\
\text { pediatria }\end{array}$ & $\begin{array}{l}\text { Sala da } \\
\text { pediatria }\end{array}$ & $\begin{array}{l}\text { Os profissionais sentam livremente } \\
\text { pela sala em cadeiras e mesas. A } \\
\text { enfermagem fica no computador } \\
\text { atualizando a planilha e conduz a } \\
\text { reunião, informando a situação do } \\
\text { paciente. Os médicos entram em algum } \\
\text { aspecto clínico e os outros profissionais } \\
\text { em alguma demanda específica. }\end{array}$ & $\begin{array}{l}\text { Diário } \\
11 \mathrm{~h}\end{array}$ & $\begin{array}{l}\text { Assistente Social, } \\
\text { Enfermeira do NIR } \\
\text { Enfermeira horizontal } \\
\text { Enfermeira plantonista } \\
\text { Fonoaudióloga } \\
\text { Médicos do NIR e pediatras }\end{array}$ \\
\hline $\begin{array}{l}\text { Kanban ou } \\
\text { Round da UTI } \\
\text { pediátrica }\end{array}$ & $\begin{array}{l}\text { Sala } \\
\text { pequena } \\
\text { na UTI }\end{array}$ & $\begin{array}{l}\text { Os profissionais em pé ou sentados } \\
\text { discutem os casos. Todos têm voz. } \\
\text { Quem conduz é a enfermagem, que } \\
\text { informa a situação do paciente em } \\
\text { relação aos procedimentos realizados } \\
\text { ou não, medicação e necessidades. } \\
\text { Os médicos falam do caso e trocam } \\
\text { informações entre eles. Os outros } \\
\text { profissionais atuam quando há } \\
\text { demanda. }\end{array}$ & $\begin{array}{l}\text { Diário } \\
11 \mathrm{~h} 30\end{array}$ & $\begin{array}{l}\text { Assistente Social, } \\
\text { Enfermeira do NIR } \\
\text { Enfermeira horizontal } \\
\text { Enfermeira plantonista } \\
\text { Fisioterapeuta } \\
\text { Fonoaudióloga } \\
\text { Nutricionista } \\
\text { Psicóloga } \\
\text { Médicos do NIR e pediatras }\end{array}$ \\
\hline $\begin{array}{l}\text { Kanban } \\
\text { multipro- } \\
\text { fissional } \\
\text { pediatria }\end{array}$ & $\begin{array}{l}\text { Brinque- } \\
\text { doteca }\end{array}$ & $\begin{array}{l}\text { Todos os profissionais da pediatria, } \\
\text { incluindo a UTI e a semintensiva. } \\
\text { Quem conduz é a enfermeira a } \\
\text { partir da planilha do censo. O clima } \\
\text { entre os profissionais é bem tranquilo, } \\
\text { com momentos de descontração. }\end{array}$ & $\begin{array}{l}\text { Semanal } \\
11 \mathrm{~h}\end{array}$ & $\begin{array}{l}\text { Assistente Social, } \\
\text { Enfermeira do NIR } \\
\text { Enfermeira horizontal } \\
\text { Enfermeira plantonista } \\
\text { Fisioterapeuta } \\
\text { Fonoaudióloga } \\
\text { Médicos do NIR e pediatras } \\
\text { Nutricionista } \\
\text { Psicóloga }\end{array}$ \\
\hline $\begin{array}{l}\text { Kanban eixo } \\
\text { verde }\end{array}$ & $\begin{array}{l}\text { Não há } \\
\text { lugar } \\
\text { fixo: } \\
\text { corredor }\end{array}$ & $\begin{array}{l}\text { Enfermeiros, médico e assistente } \\
\text { social sempre presentes. Quem } \\
\text { conduz é a enfermagem com o censo, } \\
\text { informando a situação do paciente. } \\
\text { A assistente social explicita a situação } \\
\text { social, em particular em relação à alta. }\end{array}$ & 3 vezes ao dia & $\begin{array}{l}\text { Assistente social, } \\
\text { Enfermeira do NIR } \\
\text { Enfermeira horizontal } \\
\text { Enfermeira plantonista } \\
\text { Médico do NIR } \\
\text { Médicos } \\
\text { Psicóloga } \\
\end{array}$ \\
\hline $\begin{array}{l}\text { Kanban } \\
\text { unidade de } \\
\text { internação }\end{array}$ & $\begin{array}{l}\text { Sala de } \\
\text { estudos }\end{array}$ & $\begin{array}{l}\text { Quem conduz é a enfermagem, que } \\
\text { informa a situação do paciente em } \\
\text { relação aos procedimentos realizados } \\
\text { ou não, medicação e necessidades. }\end{array}$ & Diário & $\begin{array}{l}\text { Assistente social, } \\
\text { Enfermeira do NIR } \\
\text { Enfermeira horizontal } \\
\text { Enfermeira plantonista } \\
\text { Fisioterapeuta } \\
\text { Fonoaudióloga } \\
\text { Médico do NIR e médicos } \\
\text { Nutricionista } \\
\text { Psicóloga }\end{array}$ \\
\hline $\begin{array}{l}\text { Kanban salas } \\
\text { amarela e } \\
\text { vermelha }\end{array}$ & \begin{tabular}{|l|} 
No \\
corredor \\
de \\
entrada
\end{tabular} & $\begin{array}{l}\text { Quem conduz é a enfermagem } \\
\text { diarista, que informa a situação de } \\
\text { entrada do paciente, procedimentos } \\
\text { realizados, medicação e necessidades. } \\
\text { Os médicos falam do caso, trocam } \\
\text { informações entre eles e indicam a } \\
\text { possibilidade de encaminhamento. }\end{array}$ & $\begin{array}{l}\text { Diário } \\
11 \mathrm{~h} \\
22 \mathrm{~h}\end{array}$ & $\begin{array}{l}\text { Assistente social, } \\
\text { Enfermeira do NIR } \\
\text { Enfermeira horizontal } \\
\text { Enfermeira plantonista } \\
\text { Fisioterapeuta } \\
\text { Médico do NIR } \\
\text { Médicos } \\
\text { Psicóloga }\end{array}$ \\
\hline
\end{tabular}




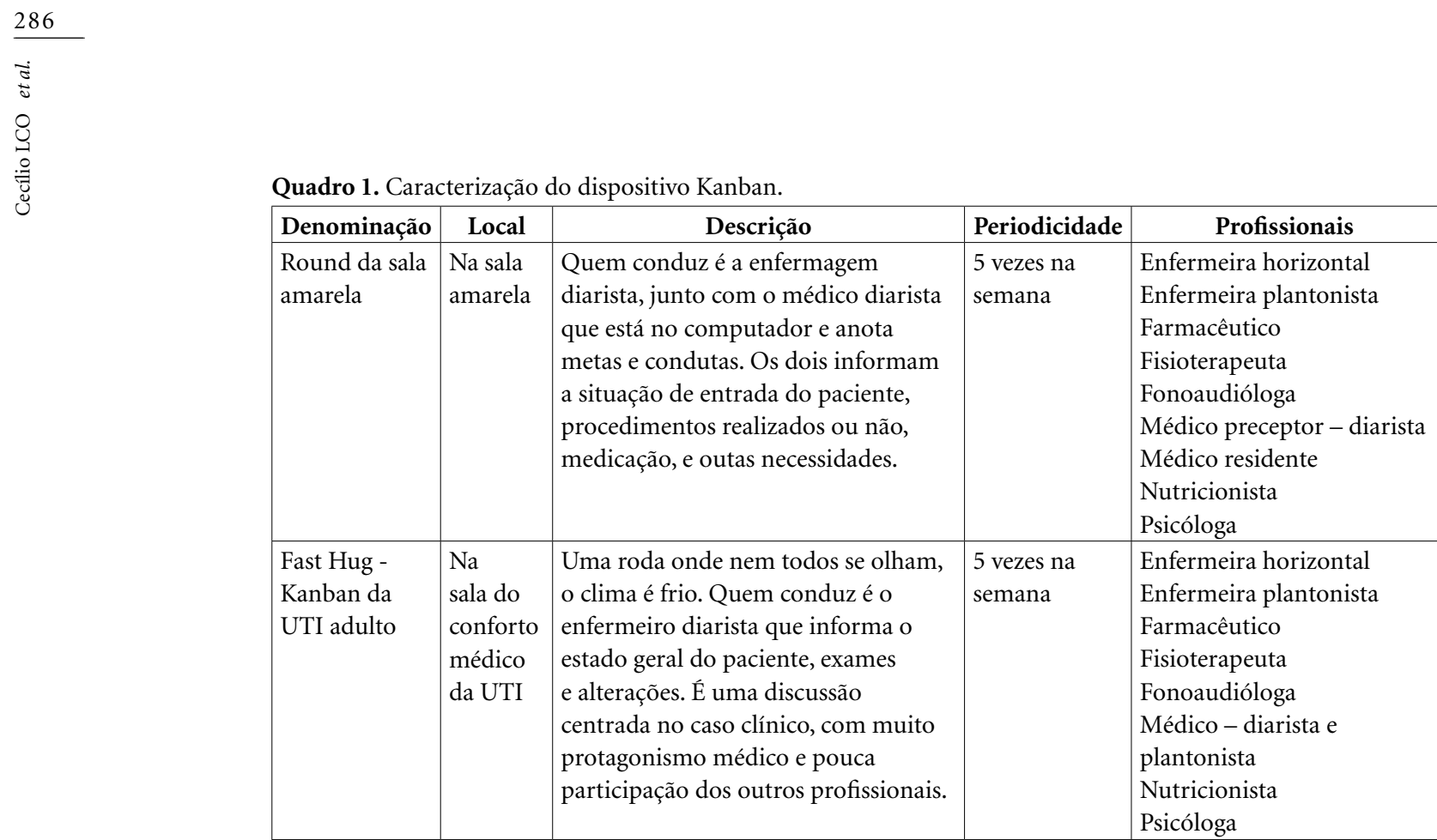

Fonte: Arranjos tecnológicos de gestão do cuidado em um Hospital de Pronto Socorro (Fapesp-PPSUS-CNPq).

ça prolongada e contínua de pesquisadores em campo para observar a operacionalização cotidiana do Kanban pelas equipes. Resultou de tal opção, uma aproximação micropolítica à gestão e produção de cuidado ${ }^{30}$, assumindo-se que esses processos se redefinem e redesenham à medida da sua utilização pelos atores institucionais.

Os diários de campo, elaborados pelos pesquisadores a cada ida ao campo, em diferentes dias e horários da semana, durante quase um ano, foram processados no coletivo de pesquisadores que se reuniu em seminários. Na primeira sistematização analítica, o material empírico foi organizado em três grandes blocos: as relações internas à equipe, as relações das equipes com os usuários e as relações da equipe com a direção do hospital. Desse material, foram selecionadas cenas para restituição para as equipes em dois seminários compartilhados (SC) dos pesquisadores com equipes do hospital. Neles, novas questões foram levantadas, tendo sido posta a necessidade de outros encontros, agora com grupos profissionais específicos (médicos, enfermeiros e não-médicos/não enfermeiros).

No Quadro 2 estão descritas as diferentes técnicas de produção de dados utilizadas. Todos os seminários compartilhados foram gravados e posteriormente transcritos, com anuência dos envolvidos, que assinaram o Termo de Consentimento Livre Esclarecido.

Para a elaboração desse artigo foram recortados dos diários de campo (DC) os registros referentes aos enfermeiros. Utilizou-se, ainda, as transcrições de seis seminários compartilhados (SC), seja daquele realizado especificamente apenas com enfermeiros, seja nos demais, quando outros profissionais faziam referências ao trabalho dos enfermeiros.

\section{Resultados}

\section{Conservação ou mudanças nas "atribuições tradicionais" da enfermagem?}

A lógica do Kanban apoia-se em dois eixos principais: a) o trabalho interprofissional para qualificar o diagnóstico e o projeto terapêutico para cada paciente (gestão clínica); b) o uso intensivo de informação atualizada sobre o paciente compartilhada pela equipe em tempo real, garantindo que todos os encaminhamentos necessários sejam agilizados, visando a máxima rotatividade e aproveitamento dos leitos (gestão de leitos). A atuação do enfermeiro é fundamental nos dois eixos. Com o correr do tempo, a relação entre gestão clínica e de leitos foi sendo alterada na dinâmica de funcionamento do Kanban, com importantes impactos no trabalho de enfermagem, como ilustra essa observação:

(...) ela estava falando de quando [o Kanban] começou. O foco era muito o cuidado e, como consequência, o gerenciamento de leitos (...) você adiantava, entre aspas, a alta desse paciente $e$ 
Quadro 2. Descrição das técnicas de pesquisa utilizadas.

\begin{tabular}{|l|l|l|}
\hline \multicolumn{1}{|c|}{ Técnica utilizada } & \multicolumn{1}{|c|}{ Atores envolvidos e local } & \multicolumn{1}{c|}{ Objetivo } \\
\hline Observação participante & $\begin{array}{l}\text { Equipes envolvidas na } \\
\text { operacionalização do kanban }\end{array}$ & $\begin{array}{l}\text { Caracterizar o funcionamento do } \\
\text { Kanban observando as relações } \\
\text { das equipes, dos profissionais e } \\
\text { usuários e os lugares }\end{array}$ \\
\hline Seminários compartilhados & $\begin{array}{l}\text { - Coordenadores da pesquisa } \\
\text { - Pesquisadores de campo } \\
\text { - Gestores do Hospital e Coordenações } \\
\text { dos eixos: verde, crítico, internação e } \\
\text { UTI adulto e criança } \\
\text { - Núcleo Regulação Interna } \\
\text { - Supervisões profissionais }\end{array}$ & $\begin{array}{l}\text { institucionais a primeira análise } \\
\text { dos achados obtidos a partir das } \\
\text { observações participantes. }\end{array}$ \\
\hline $\begin{array}{l}\text { Seminários compartilhados com } \\
\text { os Enfermeiros }\end{array}$ & $\begin{array}{l}\text { Pesquisadores e enfermeiros: gestores, } \\
\text { coordenadores de clínica, diaristas, } \\
\text { plantonistas }\end{array}$ & $\begin{array}{l}\text { Discutir com os enfermeiros } \\
\text { as questões colocadas nos } \\
\text { seminários }\end{array}$ \\
\hline
\end{tabular}

Fonte: Arranjos tecnológicos de gestão do cuidado em um Hospital de Pronto Socorro (Fapesp-PPSUS-CNPq).

otimizava o tempo dele de internação. (...) com o passar do tempo, e as salas acho que foram enchendo, e o hospital inchando (...) houve a perda da lógica da gestão clínica para a gestão pura e simples do leito, "fazer rodar". (Houve a) perda de um trabalho multidisciplinar mais compartilhado, por uma grande responsabilidade pela enfermeira diarista (Diário de Campo - DC).

Analisando os processos internos à enfermagem, o Kanban acelerou e/ou intensificou a especialização e a fragmentação da prática dos enfermeiros. Os enfermeiros dividem-se em “diaristas" e "plantonistas". Os primeiros, trabalham todos os dias úteis da semana no hospital, são "horizontais", e, em princípio, deveriam ter um contato mais próximo e contínuo com um determinado grupo de pacientes sob sua responsabilidade. Os segundos, em regime de plantão, têm contatos mais pontuais ou descontínuos com as pessoas internadas. São os diaristas, muito envolvidos com "gestão de leitos", que mais percebem o quanto tarefas de caráter mais administrativo foram, paulatinamente, ocupando mais tempo do seu trabalho cotidiano, em detrimento de contatos clínicos com o paciente.

Na época, quando a gente introduziu o Kanban (...) a gente entrava, via paciente um por um, fazia curativo, conversava com a família, criava vínculo com a família. Era outro papel, cuidar do paciente integral, para ele sair bonitinho. (...) Aí, com o tempo isso foi mudando. Então, pra focar na diminuição do tempo de permanência do paciente (...) a gente foi se perdendo. Não entrava mais na enfermaria, não vejo mais, sei mais do paciente de cabo a rabo, mas não sei da cara dele, e nem da cara do familiar, porque não tenho tempo de ir lá conversar com ele. (Enfermeiro diarista, Seminário Compartilhado - SC).

No início das observações, percebia-se uma avaliação muito positiva da enfermagem em relação à incorporação de novos arranjos tecnológicos da gestão do cuidado. Eram frequentes as manifestações de elogio e satisfação com o trabalho que vinha sendo realizado a partir da incorporação desses arranjos. O Kanban, frequentemente diziam os enfermeiros, durante as observações, "contribuiu para organizar o fluxo no/do hospital"; possibilitou "uniformizar, padronizar" as práticas; "foi um divisor de águas". Antes do Kanban "não sabíamos o que estava acontecendo com os pacientes, $o$ que tinha sido realizado, o que estavam aguardando. (...) O trabalho era muito fragmentado e desorganizado; a mudança foi muito significativa" (DC).

Portanto, o que foi ganhando maior visibilidade foram efeitos da micropolítica na operacionalização do Kanban sobre o trabalho dos enfermeiros, daí a divisão muito marcante entre os "diaristas" e os “plantonistas”. Há enfermeiros que, atuando na mesma unidade assistencial, têm práticas bastante diferentes entre si.

Atribuições de caráter puramente administrativo, que ocupam grande parte do tempo de trabalho dos diaristas, afasta-os da atividade clínica, ao ponto destes avaliarem que a sobrecarga de tais atividades comprometeria sua atuação em outras dimensões do cuidado. E, contrariamente, avaliam que o "enfermeiro plantonista" estaria mais próximo da clínica e do paciente (e do médico), por estarem desonerados das atividades administrativas cotidianas.

O enfermeiro que fica mesmo como plantonista, ele fica muito próximo, ele sabe quando o pa- 
ciente piorou, quando rebaixou, (...) que o paciente mudou. Então, ele consegue falar: tem certeza? Outras situações de alta também: você tem certeza que dá pra dar alta agora? (...) Agora, o enfermeiro plantonista, você consegue esse respeito, tem muito que são respeitados, é porque ele tem qualidade na assistência, ele tem capacidade técnica, ele sabe conduzir a equipe e mantém o seu setor em ordem (Enfermeira diarista, SC).

Portanto, é necessário reconhecer a fragmentação e especialização da prática da enfermagem consequentes à implantação do Kanban, para percebermos que diferenciações mais sutis estão ocorrendo no interior da profissão.

A gente sabia de tudo sobre o paciente, hoje em dia eu sei da tomo, do laudo, do ultrassom, mas não sei da pele e não sei do abcesso (Enfermeira diarista, SC).

Essa reestruturação do trabalho da enfermagem na dinâmica do Kanban tem produzido sobrecarga e estresse no trabalho cotidiano, como apontado principalmente pelos diaristas. Sentem-se mais cobrados, por distintos profissionais do serviço, tanto do pessoal da área administrativa quanto da equipe assistencial:

(...) A gente é cobrado por todos os lados (Enfermeiro, SC)

Aí, a gente olha os prontuários, a gente vê que tem exame aguardando, a gente tem que abrir o agendamento de todos os exames pra saber se está na agenda, se não está, reimprimir o exame, dar na mão do médico pra ele carimbar pra você de novo imprimir (...) Se tiver pendente alguma avaliação de multi, a gente tem que procurar essa equipe multi, seja médico, seja fisio, seja o que seja, e fala, olha, está aguardando uma avaliação sua, seja psico, e fala logo, já pedi avaliação, já foi avaliada, tem que estar com tudo prontinho, mastigadinho, pra aí sim chegar no gerenciamento de leito (...) (Enfermeiro, SC).

Nesse sentido, mais do que existência de novas práticas profissionais de enfermagem, verifica-se uma redistribuição de funções e responsabilidades intraprofissionais, levando à insatisfação com o trabalho, principalmente entre diaristas, e, na opinião deles, uma piora na qualidade do cuidado. Como afirmou uma enfermeira:

como responsável técnica, eu perco às vezes o sono, de pensar em como está o cuidado? De saber que tanto eu como o enfermeiro podemos responder por alguma coisa que a gente sabe que é nossa atribuição e deixou de fazer.

\section{Enfermagem e Medicina: nova interprofissionalidade ou reprodução de relações de poder?}

A enfermagem se pensa como o ápice de uma cadeia de comando e controle do corpo de enfermagem, revestida de autoridade legal-formal, de base técnica, por conta do conhecimento que adquire no correr do curso da graduação, tanto é que "sai da graduação com uma equipe de técnicos para liderar" (Enfermeira, SC). A questão da autoridade coloca-se para o enfermeiro tendo como referência o seu próprio corpo profissional. O estudo interessava-se por ver se e como os arranjos de gestão estudados resultavam em novas relações interprofissionais, em particular se haveria novas configurações de autoridade da enfermagem, muito em particular junto com os médicos.

O corpo de enfermagem, historicamente, sempre teve muita responsabilidade com o registro e produção continuada de $\operatorname{dados}^{9}$, coisa que assume centralidade e passa a ser operacionalizada com muito mais intensidade no Kanban. Se, por um lado, essa intensificação resulta em mais sobrecarga e responsabilidade no trabalho, por outro, ela poderia representar um elemento de "promoção" da enfermagem no contexto hospitalar, um incremento de seu prestígio e autoridade em relação aos demais profissionais ${ }^{31}$.

Um dos papéis centrais dos enfermeiros é coletar e organizar informações, especificamente a organização das planilhas, instrumento fundamental para viabilizar o Kanban, pois contêm as informações sobre cada paciente necessárias para a discussão e encaminhamento de providências, $o$ que já foi conceituado como correspondendo ao seu "papel de facilitador"32.

As planilhas vertebram a dinâmica da reunião do Kanban e, na medida em que os enfermeiros organizam as informações, assumem papel estratégico na gestão do leito, e essa é, com certeza, uma dimensão importante da autoridade da enfermagem hospitalar reforçada pela lógica de funcionamento do Kanban.

É que a gente acaba detendo uma maior informação a respeito de tudo que acontece (...) "O enfermeiro horizontal [ou diarista] que é o detentor da informação, o próprio enfermeiro plantonista, muitas vezes se abstém, olha, isso é com a diarista (Enfermeira, SC).

Esses profissionais acabam assumindo, em muitos momentos, o lugar de coordenação das reuniões do Kanban, o que poderia ser entendido, à primeira vista, como um reforço de sua autoridade profissional. 
A [enfermeira] comanda a reunião, apresenta todos os pacientes pelos nomes, os que chegaram hoje, conta um pouco da história de cada um, dos outros relata mais a evolução (...) (DC).

Há, portanto, o que a equipe valoriza muito e chama de "o antes do Kanban”, isso é, toda a articulação micropolítica interprofissional que antecede a reunião do Kanban, e na qual a enfermagem tem forte protagonismo, em particular os diaristas, tornando visível o seu "papel facilitador" como é possível ver nessa observação:

Pergunto como ela conduz a reunião e ela me diz: "antes de chegar aqui, faço o resumo - via prontuário-via sistema - de cada um dos pacientes. Chego na reunião com a planilha completa e com os encaminhamentos necessários pensados (DC).

Várias manifestações indicam insatisfação e conflitos na relação com os médicos. Parece que, muitas vezes, se sentem realizando as ações a serviço dos médicos, para facilitar seu trabalho. O estudo revela uma faceta do trabalho do enfermeiro (e motivo de muita insatisfação) que é desempenhar o papel de "ponte de informação", mas que poderia ser chamada de "funcionar como menino de recado" entre os profissionais da equipe multiprofissional e os médicos, ou entre os próprios médicos.

Eles não olham no prontuário a avaliação da fono. Eles perguntam pra gente o que a fono disse, se a fono não passar pra ele (Enfermeiro, SC).

(...) O cirurgião está ali do lado. Aí eu falo, é a cirurgia. Então, tem que falar com o cirurgião, e o cirurgião ali, escutando do meu lado. Mas eles não se conversam. Eu falo, doutor... é você... (Enfermeiro, SC).

De modo surpreendente, muitas vezes os enfermeiros se colocam na posição de mediadores de conflitos, em especial, entre os profissionais médicos.

[citando uma situação em que havia conflito entre os médicos] Mas ele não pediu exame? Exame? Mas o paciente é pediátrico. Por que o cirurgião tem que pedir exame? Ah, mas [foi] ele que avaliou a trauma, ele que tirou do protocolo, ele tem que pedir exame! Lá vou eu atrás do pediatra. Doutor, perguntou se o senhor não vai lá pra repetir o exame, Eu? Mas esse paciente não é meu, só fui tirar do protocolo! A mesma coisa acontece com a ortopedia (SC).

De qualquer forma, os enfermeiros relataram ter "conquistado algum poder" a partir da implementação do Kanban.

"Aqui tem o empoderamento da enfermagem", afirmou uma enfermeira para a pesquisadora. Segundo ela, isso se deve à gestão, que "banca" este lugar, que coloca as enfermeiras em espaços estratégicos de coordenação e que isso faz com que as enfermeiras (não só as coordenadoras) sejam respeitadas. Afirmam que esta "cultura" já está tão forte que não depende mais de quem (partido politico) assume a gestão (Coordenadora, SC).

(...) a enfermeira demonstrava muita segurança e autoridade na condução da reunião, conhecia detalhadamente os casos, e acionava de forma bastante tranquila e horizontal os médicos para encaminhamentos de pendências dos usuários internados (DC).

Ao mesmo tempo, e quase de forma quase paradoxal, os enfermeiros também relativizam esse empoderamento ao afirmar que o médico continua com o poder de decisão.

No Kanban, a gente não discute assistência, a gente só reporta o diagnóstico pros médicos dizerem o que vão fazer com ele. A gente dá o diagnóstico e passa a bola para o médico. Quem dá a palavra final é o médico (Enfermeiro, SC).

No entanto, consideram que em alguns contextos, e a depender da situação e do médico, conseguem compartilhar a decisão, ou, pelo menos, influenciar a decisão clínica.

Por que se a gente não tivesse esse jogo de cintura ali na hora, poderia ser que o paciente tivesse alta, porque já estava programada. Então, em várias situações, "ah, não acha melhor fazer de outra forma?" Alguns médicos aceitam essa sugestão até porque a gente está mais próximo ali (Enfermeiro, $\mathrm{SC})$.

\section{Discussão}

A adoção do Kanban no hospital estudado ilustra o processo de gerencialismo que o setor da saúde vem atravessando por todo o mundo. Sabe-se que os princípios do gerencialismo visam o reforço da gestão econômica, da eficiência e eficácia da atividade pública, não obstante suas concretizações serem variáveis entre países e serviços $^{32}$. O Kanban, em concreto, representa um mecanismo "local" (por se situar no interior do hospital) que cumpre uma dupla função associada aos princípios do gerencialismo: desfazer processos de gestão (clínica e de leitos) assentes na monoprofissionalidade e na autorregulação, e introduzir mecanismos de governança próprios às organizações, cuja eficácia se espera que decorra da adaptabilidade e flexibilidade por comparação a instrumentos de gestão externos às organizações de saúde (do tipo “top-down”). Por outras palavras, o Kanban visa reconfigurar a 
interprofissionalidade, apostando na maior partilha de informação e decisão, e alcançar maior eficácia por aproximar a tomada de decisão dos profissionais.

A consequência direta é ter que olhar para os efeitos do Kanban nas profissões de saúde. A discussão aqui apresentada centra-se na enfermagem, na medida em que reconfigurações do trabalho em saúde tendem a privilegiar análises da profissão médica ${ }^{33-36}$.

Um aspecto de discussão relevante prende-se com a bipartição das atividades tradicionais do enfermeiro entre o cuidado clínico direto ao paciente (enfermeiro plantonista) e as funções consideradas burocrático-administrativas (enfermeiro diarista). Estas últimas se referem tanto ao encaminhamento de "providências" necessárias para aumentar a rotatividade dos leitos, como à coleta $\mathrm{e}$ organização das informações que serão utilizadas nas reuniões da equipe. O enfermeiro sempre acumulou essas duas atribuições, apesar de que já se percebia, nas últimas décadas, certo debate em relação ao esmagamento do tempo dedicado ao cuidado direto ao leito, pelas responsabilidades serem mais burocrático-administrativas ${ }^{37}$. A questão é que por via do Kanban ocorre uma diferenciação intraprofissional. Esta diferenciação enquadra-se na literatura sobre os profissionais-híbridos (ou híbridos). Os híbridos são peças centrais para a concretização do gerencialismo, na medida em que levam a racionalidade gestionária para o interior das culturas e identidades profissionais, ao invés de ser imposta a partir de fora ${ }^{38}$. Não tem havido consenso no debate sobre a efetiva capacidade dos híbridos para operar as mudanças esperadas nos processos de trabalho, logo no modo de "pensar profissional". Um motivo prende-se com o grupo profissional em hibridização: genericamente médicos-híbridos reforçam o seu poder porque às competências técnico-científicas os demais médicos e profissionais reconhecem também competências de gestão ${ }^{39}$; por oposição, enfermeiros-híbridos têm dificuldade em se afirmar sobretudo na relação com demais grupos profissionais, na medida em que não lhes são reconhecidas competências para esse papel ${ }^{40,41}$, o que pode configurar papéis de gestão reativa.

Ora, os achados empíricos deste estudo parecem comprovar a resistência, senão mesmo indiferença, dos médicos ao aumento de competências burocráticas que os enfermeiros diaristas (os híbridos neste caso) adquirem no Kanban.

Os achados permitem ainda observar um outro aspeto relevante na discussão da hibridização. Os enfermeiros diaristas não alteraram a sua posição hierárquica no hospital (mobilidade vertical) em relação a outros enfermeiros e outros grupos profissionais. Isto reforça a resistência, senão mesmo indiferença de todos eles às tentativas de afirmação de controle gestionário que os enfermeiros diaristas vão reivindicando nos Kanbans. Em suma, a evidência sugere que a hibridização da enfermagem sem a alteração formal do organograma do serviço de emergência produz efeitos limitados. Por outro lado, não se sabe ao certo que efeitos são possíveis caso a alteração formal do organograma reforce o papel dos profissionais híbridos.

Outra evidência revela que o fato dos enfermeiros deterem controle oficial, e não apenas oficioso e negociado, não é necessariamente um elemento central na sua afirmação interprofissional ${ }^{37}$. O exemplo mais flagrante desta constatação é a sobrecarga de trabalho e descontentamento dos diaristas por assumirem as atividades ditas "administrativas", vivendo um afastamento ainda mais radical do cuidado direto ao paciente, atividade mais afeita aos plantonistas, por sua proximidade com os médicos e por sua desoneração de tais atividades mais rotineiras.

A responsabilização da enfermagem pela coleta, registro e sistematização de informação, em tempo real, elemento chave para o funcionamento do Kanban, dá a ela um forte protagonismo e visibilidade na condução do dispositivo, embora tal protagonismo não seja visto, pela categoria, como necessariamente um aumento de poder frente os médicos, que ainda deteriam, em boa medida, a decisão clínica e condução do caso.

Na preparação para o Kanban - "o antes do Kanban” -, quando são feitos todas as articulações necessárias para a reunião ter agilidade, o enfermeiro continua sendo insubstituível, entre outras coisas por ser o corpo profissional com mais permanência no hospital, o que mais circula e o que detém mais informações, e aí reside seu poder profissional, por vezes invisível, mas fundamental e insubstituível. E isso, podemos dizer, já era uma característica da profissão que permaneceu inalterada com o Kanban.

O que poderia ser dito é que o Kanban assume dois sentidos para a enfermagem, na medida em que dá aos enfermeiros maior protagonismo nos processos de trabalho, em concreto no gerenciamento de leitos, mas, ao mesmo tempo, os afasta dos ideais nos quais a enfermagem se profissionalizou, e considera ser a sua marca distintiva em relação à medicina. No fundo, a participação no Kanban mostra o profissionalismo de enfermagem numa encruzilhada: entre o controle buro- 
crático das organizações e a autonomização da aplicação do seu conhecimento face à medicina.

\section{Conclusões}

O Kanban tem provocado importantes mudanças na prática profissional dos enfermeiros, embora persista uma ambiguidade sobre o quanto tem havido a produção de uma nova interprofissionalidade, quando se pensa a relação com os médicos. A adoção relativamente recente do dispositivo talvez ainda não permita uma avaliação com mais alcance das possíveis transformações em curso. Entre os próprios enfermeiros, há uma ambiguidade de percepção do quanto o Kanban tem proporcionado, de fato, uma nova interprofissionalidade, na qual eles teriam uma relação mais horizontal com os médicos, influenciando mais a condução dos casos, ou estariam vivendo apenas uma exacerbação da função "secretários dos médicos", desenvolvendo principalmente atividades de suporte àqueles profissionais e a outros profissionais da equipe. O fato dos enfermeiros serem os "guardiões" da informação e desempenharem um papel crucial para viabilizar a utilização em tempo real de uma grande massa de dados clínico-operacionais, - espinha dorsal da lógica do Kanban -, sem dúvida dá a esses profissionais mais visibilidade, e o reconhecimento de sua imprescindibilidade, quando se trata de aperfeiçoar o cuidado hospitalar. Poderíamos dizer, metaforicamente, que o Kanban tem produzido pequenos tremores de terra no território da interprofissionalidade, mas não um abalo sísmico capaz de produzir uma reacomodação radical das tão arraigadas relações de poder ainda dominantes no hospital.

\section{Colaboradores}

LCO Cecílio: concepção, delineamento análise e interpretação dos dados, redação do artigo e sua revisão crítica, e aprovou a versão a ser publicada. AA Chioro dos Reis e T Correia: contribuíram para concepção, delineamento, análise e interpretação dos dados; para a redação do artigo e sua revisão crítica; leram e aprovaram a versão final do manuscrito. R Andreazza: realização da pesquisa empírica; contribuiu para a o delineamento, análise e interpretação dos dados; para a redação do artigo e sua revisão crítica. SM Spedo: contribuiu para concepção, delineamento, análise e interpretação dos dados; para a redação do artigo e sua revisão crítica. NLM Cruz e LS Barros: realização da pesquisa empírica; revisão crítica e leram e aprovaram a versão final do manuscrito. G Carapinheiro: contribuiu para a análise e interpretação dos dados; para a redação do artigo e sua revisão crítica; leu e aprovou a versão final do manuscrito. MC Schveitzer: realização de revisão crítica; leu e aprovou a versão final do manuscrito.

\section{Agradecimentos}

Às equipes do hospital estudado. A pesquisa foi financiada pela Fapesp.

\section{Referências}

1. Deslandes SF. Frágeis deuses: profissionais de emergência entre os danos da violência e a recriação da vida. Rio de Janeiro: Editora Fiocruz; 2002.

2. Magid DJ, Asplin BR, Wears RL. The quality gap: Searching for the consequences of emergency department crowding. Ann Emerg Med 2004; 44:586-588.

3. Weiss SJ, Derlet R, Arndahl J, Ernst AA, Richards J, Fernández-Frackelton $\mathrm{M}$, Schwab R, Stair TO, Vicellio P, Levy D, Brautigan M, Johnson A, Nick TG. Estimating the degree of emergency department overcrowding in Academic Medical Center: results of the National ED Overcrowding Study (NEDOCS). Acad Emerg Med 2004; 11:38-50.

4. Giglio-Jacquemot A. Urgências e emergências em saúde: perspectivas de profissionais e usuários. Rio de Janeiro: Editora Fiocruz; 2005.

5. Bittencourt RJ. A superlotação dos serviços de emergência hospitalar como evidência de baixa efetividade organizacional [tese]. Rio de Janeiro: Escola Nacional de Saúde Pública; 2010.

6. Hartz ZMA, Contandriopoulos AP. Integralidade da atenção e integração de serviços de saúde: desafios para avaliar a implantação de um "sistema sem muros". Cad Saude Publica 2004; 20:332-336.

7. Allder S, Silvester K, Walley P. Managing capacity and demand across the patient journey. Clinical Medicine 2009; 10(1):13-15.

8. Beltrammi DGM. Efetividade das intervenções para redução da superlotação nos serviços de emergência hospitalar [dissertação]. São Paulo: Instituto Sírio-Libanês de Ensino e Pesquisa; 2015.

9. Carapinheiro G. Saberes e Poderes no Hospital. Uma sociologia dos serviços hospitalares. Porto: Edições Afrontamento; 1993. 
10. Campos GWS, Amaral MA. A clínica ampliada e compartilhada, a gestão democrática e redes de atenção como referenciais teórico-operacionais para a reforma do hospital. Cien Saude Colet 2007; 12(4):849-860.

11. Chioro dos Reis AA. Entre a intenção e o ato: uma análise da política de contratualização dos hospitais de ensino (2004-2010) [tese]. São Paulo: Universidade Federal de São Paulo; 2011.

12. Brasil. Ministério da Saúde (MS). Portaria no 3.390 , de 30 de dezembro de 2013. Institui a Política Nacional de Atenção Hospitalar (PNHOSP) no âmbito do Sistema Único de Saúde (SUS), estabelecendo-se as diretrizes para a organização do componente hospitalar da Rede de atenção à Saúde (RAS). Diário Oficial da União; 2013.

13. Fleury ACC. Organização do trabalho industrial: um confronto entre teoria e realidade [tese]. São Paulo: Escola Politécnica da Universidade de São Paulo; 1978.

14. Dussauge P, Hart S, Ramanantsoa B. Strategic Technology Management. Chichester: John Wiley \& Sons; 1992.

15. Cecílio LCO, Coutinho AAP, Hamze FL, Silva AF, Batista LA, Carvalho APH. Programa SOS emergências: uma alternativa de gestão e gerência para as grandes emergências do Sistema Único de Saúde. Divulg Saude Debate Rio de Janeiro 2014; 52:202-216.

16. Scarazzatti GL. Tendências da avaliação em saúde. Debates GV Saúde 2006; 2:22-23.

17. Bandurchin A, McNally MJ, Ferguson-Paré M. Bringing back the house call: how an emergency mobile nursing service is reducing avoidable emergency department visits for residents in long-term care homes. Nurs Leadersh 2011; 24(1):59-71.

18. Wang M, Wild S, Hilfiker G, Chmiel C, Sidler P, Eichler K, Rosemann T, Senn O. Hospital-integrated general practice: a promising way to manage walk-in patients in emergency departments. J Eval Clin Pract 2014; 20(1):20-26.

19. Bittencourt RJ, Hortale VA. Intervenções para solucionar a superlotação nos serviços de emergência hospitalar: uma revisão sistemática. Cad Saude Publica 2009; 25(7):1349-1454.

20. Crane J, Noon C. The Definitive Guide to Emergency Department Operational Improvement. Boca Raton: CRC Press; 2011.

21. Liu SW, Singer SJ, Sun BC, Camargo CA. Care for Patients Boarding in the Emergency Department: Structure-Process-Outcome. Acad Emerg Med 2011; 18(4):430-435.

22. Sousa P, Mendes W, organizadores. Segurança do paciente: conhecendo os riscos nas organizações de saúde. Rio de Janeiro: Editora Fiocruz; 2014.

23. Bradley VM. Placing Emergency Department crowding on the decision agenda. J Emerg Nurs 2005; 31:247-258.

24. Chan SS, Cheung NK, Graham CA, Rainer TH. Strategies and solutions to alleviate access block and overcrowding in emergency departments. Hong Kong Med J 2015; 21(4):345-352.

25. Williams K. Advanced practitioners in emergency care: a literature review. Emerg Nurse 2017; 25(4):36-41.

26. Jennings N, Clifford S, Fox AR, O'Connell, Gardner G. The impact of nurse practitioner services on cost, quality of care, satisfaction and waiting times in the emergency department: a systematic review. Int J Nurs Stud 2015; 52(1):421-435.
27. Bristow DP, Herrick CA. Emergency department case management: the dyad team of nurse case manager and social worker improve discharge planning and patient and staff satisfaction while decreasing inappropriate admissions and costs: a literature review. Lippincotts Case Manag 2002; 7(3):121-128.

28. Albarello L, Digneffe F, Hiernaux JP, Maroy C, Ruquoy D, Saint-Georges P. Práticas e métodos de investigação em ciências sociais. Lisboa: Gradiva; 2000.

29. Poupart J, Deslauries JP, Groulx AL, Mayer R, Pires A. A pesquisa qualitativa: enfoques epistemológicos e metodológicos. Petrópolis: Editora Vozes; 2016.

30. Lapassade G. As microssociologias. Brasília: Liber Livro; 2005.

31. Carvalho T. Nova Gestão Pública e Reformas da Saúde: o profissionalismo numa encruzilhada. Lisboa: Edições Sílabo; 2009.

32. Clarke J, Newman J. The Managerial State. Londres: Sage; 1997.

33. Correia T. The Interplay between managerialism and medical professionalism in hospital organisations from the doctors' perspective: a comparison of two distinctive medical units. Health Sociology Review 2013; 22(3):255-267.

34. Currie G, Croft C. Examining hybrid nurse managers as a case of identity transition in healthcare: developing a balanced research agenda. Work, Employment and Society 2015; 29(5):855-865.

35. Jamra C, Cecílio LCO, Correia T. Os médicos e a racionalização das práticas hospitalares: novos limites para a liberdade profissional? Saude Debate 2016; 40(108):86-94.

36. Reis D, Cecílio LCO, Andreazza R, Araújo E, Correia T. Nem herói, nem vilão: elementos da prática médica na atenção básica em saúde. Cien Saude Colet 2018; 23(8):2651-2660.

37. Carvalho T. Managerialism and professional strategies: a case from nurses in Portugal. $J$ Health Organ Manag 2012; 26(4):524-541.

38. Llewellyn S. 'Two-way windows': clinicians as medical managers. Organ Studies 2001; 22(4):593-623.

39. Correia T, Denis JL. Hybrid management, organizational configuration, and medical professionalism: evidence from the establishment of a clinical directorate in Portugal. BMC Health Services Research 2016; 16(Supl. 2):161.

40. Apesoa-Varano EC. Educated caring: the emergence of professional identity among nurses. Qualitative Sociology 2007; 30(3):249-274.

41. Nugus P, Greenfield D, Travaglia J, Westbrook J, Braithwaite J. How and where clinicians exercise power: interprofessional relations in health care. Soc Sci Med 2010; 71(5):898-909.

Artigo apresentado em 23/04/2019

Aprovado em 20/08/2019

Versão final apresentada em 30/09/2019 